\title{
La pedagogía: el complemento estratégico de la Educación Ambiental ${ }^{1}$
}

\author{
Marcela Andrea Moscoso Casallas \\ Universidad Minuto de Dios, Ubaté, Colombia \\ marandremc@gmail.com \\ Vilma Aurora Garzón Guerrero² \\ Universidad Minuto de Dios, Ubaté, Colombia \\ alondravag_10@hotmail.com
}

1 Artículo de divulgación.

2 Estudiantes de décimo semestre de Licenciatura en Pedagogía infantil de la Corporación Universitaria Minuto de Dios - UNIMINUTO. 


\title{
La pedagogía: el complemento estratégico de la Educación Ambiental
}

\section{Resumen}

Este artículo parte de un proceso de investigación de carácter cualitativo y documental, bajo un enfoque hermenéutico y sociocrítico, con el cual se buscó analizar la importancia, funcionalidad, relación y pautas para la selección y aplicación de estrategias pedagógicas dentro de la Educación Ambiental. Se identificó que este tipo de educación se ha caracterizado por una serie de intenciones que se han visto limitadas por sus pocas estrategias, pese al hecho de ser interdisciplinar y transversal. Tal situación se evidenció en la Institución Educativa Departamental Nuestra Señora del Carmen, sede General Santander, del municipio de Lenguazaque, Cundinamarca. Por lo cual, se propuso como solución la formulación de una cartilla o catálogo didáctico en la que se plantearon una serie de estrategias, recomendaciones y pautas para el desarrollo didáctico de la Educación Ambiental en el aula, desde un proceso vivencial, reflexivo, conceptual, dinámico, participativo, creativo e innovador.

Palabras clave: Educación Ambiental, estrategias pedagógicas, didáctica ambiental, Pedagogía Ambiental, 3R.

\section{Pedagogy: A Strategic Complement for Environmental Education}

\begin{abstract}
A qualitative-based and documental investigation process was used in the present research, this includes a sociocritic and hermeneutic approach to analyze the importance, functionality, relation and standards in the selection and implementation of pedagogical strategies for environmental-related education. Environmental-related education has been historically limited in its interests by wrong approach strategies, although its transversality and interdisciplinarity. The investigation was carried out at Institución Educativa Departamental Nuestra Señora del Carmen, General Santander, Lenguazaque, Cundinamarca municipality. As a way to solve the above described issue, a didactic booklet was designed. In such booklet, a series of strategies, recommendations and standards for the didactic development of environment-related education in the classroom -taking into account aspects such as experiential, reflective, conceptual, dynamic, creative and innovative processes-
\end{abstract}

Keywords: Environment-related education, Pedagogical strategies, Environmental didactics, Environmental pedagogy, $3 R$.

\section{A pedagogia: o complemento estratégico da Educação Ambiental}

\section{Resumo}

Este artigo parte de um processo de investigação de caráter qualitativo e documentário, baixo um enfoque hermenêutico e socio crítico, com o qual se procurou analisar a importância, funcionalidade, relação e pautas para a seleção e aplicativo de estratégias pedagógicas dentro da Educação Ambiental. Identificou-se que este tipo de educação se caracterizou por uma série de intenções que se viram limitadas por suas poucas estratégias, pese ao fato de ser interdisciplinar e transversal. Tal situação se evidenció na Instituição Educativa Departamental Nossa Senhora do Carmen, sede Geral Santander, do município de Lenguazaque, Cundinamarca. Pelo qual, se propôs como solução a formulação de uma cartilla ou catálogo didático na que se propuseram uma série de estratégias, recomendações e padrões para o desenvolvimento didático da Educação Ambiental no sala, desde um processo vivencial, reflexivo, conceitual, dinâmico, participativo, criativo e inovador.

Palavras chave: Educação Ambiental, estratégias pedagógicas, didática ambiental, Pedagogia Ambiental, 3R. 


\section{Introducción}

La educación es vista como una disciplina que unifica saberes, experiencias, componentes pedagógicos y didácticos que están al servicio de la formación integral del ser humano. Como tal, el docente y todo agente educativo tienen una responsabilidad social y pedagógica determinante dentro de la formación de una sociedad sustentable. Pero, ¿qué relación existe entre educación y medio ambiente? La pregunta se plantea toda vez que el medio ambiente constituye todo el entorno natural que nos rodea y, además, es el hábitat natural de cerca de millones de especies tanto de fauna y flora como de seres humanos de toda edad, condición y características.

La respuesta es sencilla pero, en ocasiones, es compleja llevarla a un contexto práctico. Primero, es sencilla porque la educación es de cierto modo la principal estrategia para la formación y generación de cambio de actitudes, saberes, experiencias, vivencias y conductas en el ser humano, en pro de una transformación tanto personal como colectiva dentro de una formación ambiental. Segundo, su aplicación es compleja, pues pese al hecho de los objetivos de la Educación Ambiental, muchas veces no existen las estrategias pertinentes para llevarla a cabo dentro de la educación formal e informal.

Empero, vale preguntar, ¿qué son las estrategias pedagógicas dentro del contexto de la Educación Ambiental? Las estrategias pedagógicas son acciones que se manejan dentro de un proceso de enseñanza-aprendizaje que tiene una finalidad o intención pedagógica, constituyendo así las herramientas teóricas y prácticas que permiten fortalecer, desarrollar y promover una Educación Ambiental, la cual: «Busca formar sujetos en la multidimensionalidad de su ser... capaces de hacer una lectura compleja del ambiente y proponer acciones complejas sobre el ambiente, para su transformación»(Tovar-Gálvez, 2013,p. 888). Pero, más aún, es necesario tener en cuenta que la educación es un proceso dinámico y cambiante que siempre debe partir del contexto y de las necesidades reales del educando y del medio en que se desarrolla.

En ese orden de ideas, la Educación Ambiental deja de ser una mera intención para convertirse en un componente estratégico de transformación; es ahí donde la pedagogía juega un papel importante como articulador y generador de intenciones fundamentadas y puntuales en torno a los objetivos propuestos dentro del proceso de enseñanza- aprendizaje.

Sin duda, las estrategias pedagógicas son determinantes en el proceso de enseñanza-aprendizaje, sin embargo, se ha observado que uno de los mayores 
problemas que ha tenido la Educación Ambiental ha sido la falta de claridad de las estrategias, en términos de selección y aplicación en un contexto educativo. En ocasiones se piensa que simplemente con la siembra de un árbol o con la ubicación de una secuencia de canecas en diferentes áreas del colegio se está promoviendo una Educación Ambiental, tal vez se haga, pero de una forma superficial y efímera.

Es entonces cuando surge la necesidad de buscar una forma de atender este limitante, para lo cual es pertinente aclarar que, quizás, uno de los promotores de este tipo de errores es el docente, pues algunas veces él no tiene claridad al momento de aplicar o diseñar una serie de estrategias que permitan realmente formar seres humanos capaces de entender su relación y responsabilidad para con el cuidado y conservación del medio ambiente, lo que restringe el proceso formativo del educando. Después de todo, el docente también cumple un rol como diseñador de estrategias y mediador de aprendizajes, ya que es el encargado de dar tales intenciones pedagógicas a sus acciones, recursos o medios que se utilicen dentro del proceso de formación ambiental.

Es interesante examinar esta situación a partir de un contexto más práctico. Para cumplir ese fin se estudiará un caso del municipio de Lenguazaque, Cundinamarca, una región que se caracteriza por una amplia diversidad de campos verdes, una población amigable y unos niños que poco a poco intentan transformar la sociedad para desarrollar una más sustentable. Con respecto a esto es necesario decir que el interés en los niños está, pero muchas veces las estrategias son muy «pobres e intermitentes», lo cual ha generado que ciertos aprendizajes y determinados hábitos sean mal direccionados, que no exista un adecuado proceso de acompañamiento pedagógico o simplemente se pasen por alto.

Esta es una situación frecuente, más aún cuando hablamos de una sociedad cambiante, pues, al ser dinámica, se hace a su vez desafiante para la educación y sus agentes educadores, lo que exige un cambio de paradigmas educativos a partir del contexto.

Retomando la idea de la revisión del contexto práctico, en la Institución Educativa Departamental Nuestra Señora del Carmen, sede General Santander, del municipio de Lenguazaque, en los estudiantes de tercero, cuarto y quinto de primaria, se observó reiteradas veces que las estrategias pedagógicas que manejan las docentes están más enmarcadas en cuestiones estándar como la elaboración de jardines, exposiciones y campañas; sin embargo, en ocasiones, cuando el docente aplica la estrategia pedagógica, no existe una completa claridad de los objetivos propuestos o los mismos educandos no saben de forma clara por qué lo hacen y cuál es la finalidad de eso, es decir, existe una intención pero con unos objetivos superficiales y unas estrategias un tanto planas o lineales - muy poco dinámicas-, por lo que se dificulta el desarrollo de aprendizajes significativos en el educando en 
cuanto a los temas de Educación Ambiental. Asimismo, no existe amplia variedad estratégica, generando así desmotivación y monotonía dentro del proceso formativo del educando.

Analizar y establecer una propuesta que responda a esta necesidad, dentro del área de Educación Ambiental y sus componentes estratégicos, pedagógicamente hablando, es fundamental, pues de ello dependerá el éxito de un proceso de enseñanza-aprendizaje para las nuevas generaciones, lo cual trae consigo beneficios de tipo social, cultural, humano, natural y formativo.

De esta forma, este estudio, de carácter documental y cualitativo, permite ver lo importante que es desarrollar estrategias pertinentes, claras, dinámicas, interdisciplinares, motivantes, significativas, creativas, participativas y variadas dentro de la aplicación de la Educación Ambiental, esto con el propósito de formar seres humanos con cultura ambiental, comprometidos y con capacidad de construir experiencias y saberes que promuevan el cuidado y la conservación del medio ambiente.

Es menester, pues, innovar en el aula de clase y dejar de ver la Educación Ambiental simplemente como una acumulación de información y de buenas intenciones, de tal manera que se aplique un componente más crítico, significativo, intersubjetivo y relacional, partiendo siempre del contexto real del medio y del niño.

De acuerdo con esto, el objetivo general es fortalecer la Educación Ambiental desde el uso estratégico de técnicas y recursos didácticos que promuevan un aprendizaje significativo, creativo y participativo a partir de una revisión teórica. Para desarrollar tal fin se propone una cartilla o catálogo didáctico de estrategias, pautas y recomendaciones de aplicación ambiental y pedagógica, útiles para el docente dentro de su praxis pedagógica y su acto didáctico. Se aplican las 3R reducción, reciclaje y reutilización- dentro del contexto educativo, pero como ejes de fundamentación pedagógica: la reducción hace referencia al ser más selectivos con los contenidos y estrategias que se manejan, reduciendo o retirando aquellas que realmente no sean necesarias; el reciclaje y la reutilización parten de dos líneas de acción, una es vista como material didáctico y otra se enfoca en darle el mayor uso posible a los recursos y estrategias con que contamos dentro del aula de clase o los espacios educativos, desde su adaptación y contextualización.

Así las cosas, se busca que la comunidad siempre sea un participante activo y dinámico en la atención integral al medio ambiente. Asimismo, se pretende aportar al docente una fuente de innovación, adaptación y articulación de saberes y técnicas que le permitan desarrollar integralmente a sus educandos y fortalecer el cultivo de actitudes, acciones, saberes y conductas amigables y comprometidas con el medio ambiente y todo lo que lo compone. 
Por tanto, la Educación Ambiental debe fortalecerse desde la aplicación y diseño de estrategias pedagógicas pertinentes y coherentes que posibiliten desarrollar un aprendizaje multidimensional e interdisciplinar en el educando; entonces, el docente debe demostrar su flexibilidad dentro de su praxis pedagógica, pues es el encargado de aplicar esa intención pedagógica a los recursos, medios, técnicas o estrategias didácticas y pedagógicas que constituyan la formación de un aprendizaje significativo y funcional en sus educandos, recursos que además promuevan el cuidado del medio ambiente desde sus experiencias, saberes e intereses. Así, se busca responder el siguiente interrogante: ¿qué debe tener en cuenta el docente a la hora de aplicar y diseñar estrategias pedagógicas significativas dentro de la Educación Ambiental?

\section{Marco teórico}

La Educación Ambiental es un eje dinamizador que sirve para modificar las actitudes de las personas, de manera que éstas sean capaces de evaluar los problemas de desarrollo sostenible o sustentable y abordarlos, es decir:

[...] es un proceso que reconoce valores y aclarar conceptos centrados en fomentar las actitudes, destrezas, habilidades y aptitudes necesarias para comprender y apreciar las interrelaciones entre el ser humano, su cultura y la interrelación con la naturaleza[...] es fundamental para adquirir conciencia, valores, técnicas y comportamientos ecológicos y éticos [...] es un proceso, democrático, dinámico y participativo (Rengifo, Quitiaquez y Mora, 2012, p. 4).

Asimismo, según Novo (como se cita en Rengifo et al., 2012, p.5): «La Educación Ambiental no debe verse ni como una asignatura, ni como un conjunto de actividades, sino como un acercamiento globalizador e integrador de la realidad». Por consiguiente, la Educación Ambiental tiene que atender a la formación:

[...] y a la construcción de un tipo de conocimiento que involucre la crítica y la capacidad imaginativa, de indagación, propositiva y de transformación de las relaciones del humano consigo mismo [...] y con los micro espacios de la vida cotidiana hasta los sistemas culturales, sociales, políticos y económicos más amplios —estatales, interestatales y globales_y de la articulación de estas construcciones sociales con los procesos naturales (Ceballos, 2013, pp. 9-10).

De esta manera, la Educación Ambiental -EA- constituye una herramienta estratégica para evaluar el proceso entorno a los problemas para el desarrollo de una sociedad sustentable. Es un proceso constante, dinámico, que mueve masas, que busca transformar realidades, que parte del reconocimiento de valores, actitudes, saberes, experiencias, vivencias, destrezas y habilidades que desarrolla el ser humano o que presenta frente a su relación con el medio natural, relación que se evidencia y refleja en cada campo y área en que se desenvuelve como sujeto sociocultural, ético y formativo. Del mismo modo, la EA debe nacer del contexto cotidiano del sujeto, donde no se convierta en una estructura de actividades sino en un integrado de la 
realidad, pues se parte del contexto propio e inmediato del niño, joven o adulto para que, posteriormente, el sujeto trascienda a otras áreas de acción y de contexto más regional y complejo. La Educación Ambiental debe integrar las capacidades propositivas, de crítica, análisis, reflexión, indagación, exploración y transformación del ser humano a favor del fortalecimiento del comportamiento y de los saberes ecológicos, es decir, una educación que esté a disposición de la interrelación entre el hombre y la naturaleza.

Esasícomo la EA «es un tipo deeducación caracterizada por la interdisciplinariedad, el encadenamiento de causas y efectos, el sentido global, el internacionalismo, el planteamiento de una nueva ética y acción» (Rodríguez de Moreno, 2004, p.2); es, además, transversal, debe impregnar toda la acción educativa y constituye una responsabilidad total de la comunidad educativa (Rengifo et al., 2012). Según Ibis, la transversalidad es: " [...] el conjunto de características que distinguen a un modelo curricular cuyos contenidos y propósitos de aprendizaje van más allá de los espacios disciplinares y temáticos tradicionales» (como se cita en Rengifo et al., 2012, p. 6); por ese motivo es necesario que los «procesos educativos se estructuren de acuerdo a los contextos ambientales particulares, por lo que son flexibles; se busca hacer integración de saberes y experiencias, de tal manera que se construya esa postura compleja» (Tovar-Gálvez, 2013, p.888).

Para tal propósito, «la Educación Ambiental debe ser entendida como un proceso dinámico y participativo, orientado a la formación de personas críticas y reflexivas con capacidades para comprender las problemáticas de sus contextos» (Ley 1549, 2012).

Asípues, la Educación Ambiental se concibe como una educación interdisciplinaria — combina diferentes áreas de trabajo o se puede desarrollar desde diferentes áreas académicas - , tiene un sentido global — parte de un contexto inmediato y busca trascender, por ello comprende y se define el entorno como un sistema complejo e interdependiente-, es internacionalizada — tiene un propósito, objetivo e intención definida-, transversal — busca una acción, compromiso y corresponsabilidad por parte de toda la comunidad educativa en que se desarrolle-, es estructurante — debe ser organizada, objetiva, integradora de saberes y experiencias, parte siempre del contexto real de la persona como agente de cambio y propositiva-, es participativa y dinámica, y su finalidad radica en formar seres humanos críticos, reflexivos y transformadores de realidades, para tal fin «integra el principio de equidad ambiental»(Echarri, 2009, p.10). Por lo cual, la Educación Ambiental es política: genera repercusiones de carácter social y cultural. Dentro de ese orden de ideas, surge una característica más de la Educación Ambiental pues ésta debe orientarse a la resolución de los problemas concretos del medio humano; estar orientada hacia el futuro y fundamentarse en la investigación científica y educativa, tal como lo indica la Conferencia Intergubernamental sobre Educación Ambiental: de Tbilisi —uRSS—, 
Informe finalde 1977 (Echarri, 2009).

Todavía más, en la EA es necesario aprender sobre el ambiente, aprender en el ambiente y aprender para el ambiente, canalizarla como una educación para el desarrollo sostenible (Rengifo et al., 2012). Según Orduna (2005), la meta de la Educación Ambiental es: «Aprender a aprender cómo utilizar y relacionarse con los recursos naturales para mejorar las condiciones de vida actuales, preservando esos recursos para el desarrollo de generaciones futuras» (Como se cita en Echarri, 2009, p. 35); donde la Educación Ambiental tiene un carácter humanista e integral y no se reduce a un aprendizaje de contenidos determinados, sino que:

Supone una apertura de mentalidad, de actitudes, de formas de entender las cosas, de maneras de actuar y decidir, de hábitos, de modos de participar. [...] debe impartirse a personas de todas las edades, a todos los niveles y en el marco de la educación formal, no formal e informal. [...] Proponiendo una acción social (2009, pp. 36-37).

Por esta razón, podemos decir que la Educación Ambiental es un tema de interés social, aplicado dentro de un contexto pedagógico, didáctico y educativo, con fines sustentables que se pueden lograr mediante un proceso de enseñanza y aprendizaje integral sin discriminación alguna.

Con esto en mente, es significativo hablar sobre la Pedagogía Ambiental y su relación con la Educación Ambiental. Para ello, primero se debe partir por definir el término Pedagogía Ambiental. Esta pedagogía «concibe y estructura los procesos educativos en función de lograr conciencia o cambio de actitudes en los sujetos, para que éstos le den mayor valor a lo natural y se comprometan con su cuidado o conservación» (Tovar-Gálvez, 2013, p. 886); se orienta a una fusión con los criterios básicos de la cooperación, de la ayuda mutua, de la solidaridad entre los seres humanos y la naturaleza; de manera flexible, creativa, innovadora, con la mayor apertura hacia las expresiones múltiples de las culturas, en asocio permanente y comprometido con la diversidad natural. Tiene como desafío la construcción de una cultura ambiental, instalando la capacidad de toma de conciencia y de reconstrucción de hábitos, valores, intereses, perspectivas, estrategias y acciones para habitar y relacionarse desde compromisos solidarios y responsabilidades entre los seres humanos por todos los seres humanos (Ceballos, 2013).Es ahí donde se observa el desarrollo en una sociedad auto sostenible, por esto la Educación Ambiental no puede ser vista como un proceso lineal unidireccional sino como una construcción social, constante y permanente.

Esto quiere decir que la Pedagogía Ambiental complementa la Educación Ambiental, ya que puede integrar tres etapas: educación sobre el medio, cuando plantea los contenidos; educación a través del medio, en lo relacionado con la 
metodología; y la educación a favor del medio al proponer los objetivos y los valores a lograr (Rodríguez de Moreno, 2004). Estas etapas se ven fortalecidas a partir de la etapa crítica del proceso pedagógico, en la que se parte de lo simple a lo complejo y supone ir desaprehendiendo lo aprendido, para que, de esa manera, haya realmente una construcción del conocimiento local y sustentable; aquí lo importante es la compatibilidad entre la evolución humano-natural y la sociocultural, desaprender el racionalismo instrumental y construir una racionalidad ambiental (Ceballos, 2013). Por tanto, "la educación [...] implica aprender y desaprender constantemente» (Manrique y Gallego, 2013, 102).

Pero no se puede olvidar que «quienes orientan los procesos educativos son quienes facilitan la aproximación del sujeto a lo natural y son quienes disponen recursos y saberes para los aprendizajes» (Tovar-Gálvez, 2013, p. 887), dado que la EA busca integrar sus objetivos y métodos en el sistema educativo formal, pero para lograrlo necesita de métodos pedagógicos que lo posibiliten. Debido a que a lo largo de la historia la educación ha sido vista como «una teoría con buenas intenciones y malas estrategias» (Echarri, 2009), tal y como lo señala el Libro Blanco de la Educación Ambiental en España, la Unesco propone recurrir a un nuevo paradigma educativo en el que encajaría perfectamente la teoría del aprendizaje significativo: «Para llegar a una buena gestión del medio ambiente, no basta la simple información. Es preciso modificar los enfoques, actitudes y comportamientos humanos y adquirir nuevos conocimientos, y todo ello depende en gran medida de la educación»(Como se cita en Echarri, 2009, p. 69).

Recapitulemos un poco. La Educación Ambiental es una estrategia que busca generar un cambio y transformación en la sociedad, hacerla partícipe activa del cuidado, conservación y protección del medio ambiente, a partir de un cambio actitudinal y un proceso dinámico, integrador y humanista. La Educación Ambiental es interdisciplinar y transversal, posee una intención sustentable, transformadora, critica y multidireccional, pues no se limita a un sólo contexto o área de acción, sino que parte de un nivel simple o local aún más complejo, por eso es necesario que siempre inicie del contexto real e inmediato del niño, donde se busque su desarrollo como sujeto social, cooperativo, dinámico, reflexivo, participativo, innovador y creativo. Para tal propósito es necesario desaprender lo aprendido y generar un cambio real mediante la construcción de una racionalidad ambiental, en la que la Pedagogía Ambiental constituya la estructura de los procesos educativos que enmarcan el desarrollo de una conciencia y el cambio de actitudes hacia el medio ambiente, que se van completando con la ética ambiental y las prácticas amigables con el medio. No obstante, a lo largo del tiempo se han ido presentado algunos limitantes a la hora de articular y aplicar la educación y la Pedagogía Ambiental, uno de ellos ha sido las pocas estrategias aplicadas en esta área, razón por la cual es fundamental generar un cambio en el paradigma educativo, surgiendo así el aprendizaje significativo y constructivo como modelo apropiado para lograr las diferentes metas, intenciones o propósitos de la Educación Ambiental. 
Para ello, Novak presenta la Teoría de la educación como una herramienta pedagógica que promueve los conocimientos, destrezas, valores y actitudes planteados por la Educación Ambiental_EA-. Allí se destaca el papel activo del aprendiz, quien se responsabiliza de su propio proceso pedagógico, y propone la eficacia de comprometerse también mediante el componente emocional. Su hipótesis sostiene que se consigue un aprendizaje más significativo cuando se integra el factor emocional, pues así será más fácil promover los cambios en las actitudes propuestos por la EA, impulsando, como dice Gowin, una educación que integre «pensamiento, sentimiento y acción» (como se cita en Echarri, 2009, p. 70).

Todo parece confirmar la necesidad de aplicar o desarrollar una didáctica ambiental en el aula, correspondiente a esas estrategias, técnicas, recursos o medios que se utilicen de forma significativa y multifuncional dentro del proceso formativo del educando, estrategias que promuevan o permitan un cambio de actitudes, el desarrollo y fortalecimiento de saberes y experiencias significativas con el medio ambiente, y que, a su vez, busquen formar sujetos activos, críticos, conscientes, sensibles, éticos, propositivos, innovadores, creativos y reflexivos a la hora de hablar, convivir y relacionarse con el medio natural que les rodea.

Ahora bien, ¿cuáles son las características de la didáctica ambiental?, según Tovar-Gálvez (2009), ésta debe «centrarse principalmente en la experiencia; busca la motivación y la atención de los sujetos hacia los temas a tratar; las actividades son múltiples y no necesariamente están articuladas u organizadas según un fundamento teórico sobre la enseñanza-aprendizaje-evaluación» (p. 889). Además de esto, podemos citar algunas consideraciones a tener en cuenta. En primer lugar, de acuerdo a Dimopoulos, «los procesos educativos que se desarrollan en espacios alternativos a la escuela, en los que los sujetos tendrán contacto directo con aquello que deben preservar» (como se cita en Tovar-Gálvez, 2013, p. 887); a su vez los materiales didácticos propician una educación más dinámica y eficaz, y conllevan una transmisión de conocimientos (Manrique y Gallego, 2013). El estudiante interactúa de manera más práctica y lúdica con los saberes. Novo (1995) sostiene que los programas y las actividades de la Educación Ambiental no sólo deben permitir que los estudiantes comprendan cuáles son los problemas del medio ambiente-contaminación, degradación de suelos y ecosistemas—, sino por qué han surgido y cómo deben ser controlados.

La Educación Ambiental, de acuerdo a Orduna: "Utiliza como metodología fundamental la educación en el medio vivencial - tanto en la educación formal, como en la no formal o en la informal-, a través de metodologías activas y participativas (como se cita en Echarri, 2009, p. 38). García y Cubero dicen que la razón del escaso desarrollo del ámbito didáctico en Educación Ambiental obedece a la débil implantación de la perspectiva constructivista en educación ambiental, de ahí «[...] la necesidad de aplicar una "metodología constructivista"» (como se cita en 
Díaz, Castillo y Díaz, 2014, p. 59); los contenidos de la Educación Ambiental deben programarse atendiendo a cada cultura y tiempo específico. Así, la presentación de situaciones reales conocidas por el alumno durante el aprendizaje le permite acercarse más y más tanto a la propia realidad como a lo que se intenta enseñar. (Echarri, 2009)

También se debe considerar que existen unos principios metodológicos, los cuales son: propiciar el mayor grado posible de contacto con la realidad, favorecer la explicación de las ideas y representaciones de los alumnos, partir de los problemas medioambientales, potenciar la interdisciplinaridad e integración de estudios, crear situaciones de aprendizaje motivadoras, usar estrategias de investigación, diversificar al máximo los recursos, facilitar el trabajo en equipo, potenciar la interacción comunicativa entre alumnos y profesorado, favorecer la elaboración y comunicación de conclusiones y propuestas de actuación, incentivar el trabajo en equipo entre el profesorado así como la investigación-acción (García y Galiano, 2003).

En pocas palabras, las estrategias, la metodología o la didáctica ambiental debe ser vivencial - para permitir un contacto directo con la naturaleza-, variada, tanto en sus recursos como en sus actividades; lúdico-participativa; motivadora, contextualizada, activa y dinámica; teórico-práctica, constructivista y significativa; interdisciplinar, investigadora y comunicativa. Estos aspectos se deben fortalecer mediante el uso integral de materiales didácticos con una intención pedagógica clara y definida, que le permita al educando interactuar de forma práctica, lúdica y estimulante.

Ahora es oportuno hablar sobre algunas estrategias o técnicas que permiten desarrollar y llevar a cabo, de forma eficaz, una Educación Ambiental significativa, creativa y participativa.

En primer lugar, tal y como lo indica Tovar-Gálvez (2013), la didáctica ambiental centrada en la resolución de problemas, estudio de casos y desarrollo de talleres, se debe caracterizar por integrar las diferentes dimensiones del saber en la resolución del problema, incluyendo la dimensión experimental en el marco de la solución del problema.

Estas estrategias deben estar centradas en unos temas o problemas ambientales, lo cual implicará una serie de tareas de investigación; para ello se podrían utilizar metodologías sociales, en las que se incluya la discusión grupal, con el fin de generar reflexión y compromisos para el cambio, pues:

[...] los debates y discusiones los cuales permiten la comunicación de experiencias, ideas, preconceptos, vivencias, mediante el lenguaje, obliga a todos los participantes de una manera espontánea y familiar a dar su opinión, a formular ideas y a proponer soluciones [...] (Rengifo et al, 2012, p. 11). 
Presentándose así un aprendizaje colaborativo en la cual los estudiantes resuelven problemas, responden preguntas, discuten, explican, debaten. Asimismo, según Mayer (2004), estrategias como la resolución de problemas desarrollan aprendizajes significativos en el educando (como se cita en Echarri, 2009).

Es pertinente agregar que los talleres también son excelentes herramientas dentro de la Educación Ambiental pues, tal como lo señala Arango (1996), ésta es una «modalidad de trabajo que se plantea como alternativa educativa especialmente cuando se reconoce que la base del desarrollo humano es la participación, la creatividad y la autonomía» (como se cita en Rengifo et al., 2012, p.12), aspecto que enriquecerá y fortalecerá la EA dentro del aula de clase.

En segundo lugar, encontramos el juego, el cual, según Torres (2002), más que una estrategia pedagógica, es:

Un elemento primordial en las estrategias para facilitar el aprendizaje, se considera como un conjunto de actividades agradables, cortas, divertidas, con reglas que permiten el fortalecimiento de los valores: respeto, tolerancia grupal e intergrupal, responsabilidad, solidaridad, confianza en sí mismo, seguridad, [...] y facilitan el esfuerzo para internalizar los conocimientos de manera significativa" (Como se cita en Cálad, 2013, p. 118).

Más aún, Barraza (1998) argumenta que: «El juego es un importante medio para explorar y descubrir el ambiente, a través de él se puede incrementar el interés por lo que nos rodea, [...] y estimular la imaginación, permitiendo el desarrollo de un aprendizaje divertido» (como se cita en: Díaz et al., 2014, pp. 50-51). El cual, además, puede ser significativo y representativo para el educando.

En tercer lugar, están estrategias como el trabajo de campo, el cual proporciona al estudiante participante una experiencia directa con su medio ambiente: « [...] las campañas ecológicas, las cuales se programan para generar actitudes, hábitos y valores [...] los grupos ecológicos [...] proyectos, campañas, salidas de campo, actividades lúdicas de reflexión sobre el ambiente entre otros» (Rengifo et al., 2012, p.12). Estas estrategias fortalecen y desarrollan habilidades, actitudes, destrezas, conocimientos y vínculos en el educando con el medio ambiente que lo rodea. Pero, a su vez, existen otras estrategias tales como: ferias ecológicas, visitas a espacios ambientales, huertas, experimentación, actividades artísticas y plásticas —pintura, escultura, gráfica-, y los conversatorios (Cálad, 2013).

En cuarto lugar, se maneja el arte, la literatura y la exploración del medio como bases estratégicas dentro de la educación y la didáctica ambiental. En cuanto al arte, Díaz et al. (2014) arguye que es de gran importancia, ya que hace posible el encuentro entre los conocimientos y la emoción y entre el significante y el significado, 
convirtiéndolo en un escenario para el encuentro de lenguajes. Este mismo autor, citando a Prieto, sostiene que los cuentos deben estimular la imaginación y el interés en los niños para hacerles pensar y decidir, por ello deben tener la capacidad de llenar de ilusión, pero a la vez de conocimientos, esto en cuanto a la parte literaria. Ahora bien, en cuanto a la exploración del medio, éste le permitirá al niño descubrir cómo funcionan las cosas que hay a su alrededor, produciendo en él emociones de curiosidad y sorpresa (2014); esta última genera esa metodología vivencial que tanto requiere la Educación Ambiental en la actualidad.

A nivel de técnicas tenemos el uso de los mapas conceptuales, fundamentales desde la teoría de la educación de Novak (1977, 1990, 1998) y la teoría del aprendizaje significativo de Ausubel (1968), en la que se consideran los mapas conceptuales como una herramienta pedagógica:«[...] de representación del conocimiento»( Como se cita en Echarri, 2009, p. 64) que, en general, se utilizan para conseguir un aprendizaje más significativo, ayudando a sistematizar y estructurar la información. Por ende, los mapas conceptuales son excelentes estrategias para desarrollar aprendizajes significativos dentro de la Educación Ambiental.

Otras técnicas (Mayer, 2004) que cabe mencionar son las siguientes: dar retroalimentación productiva al educando, dar actividad, concreción y familiaridad, explicar con ejemplos, fomentar las estrategias de aprendizaje — debe aprender a aprender-, fomentar estrategias de resolución de problemas, crear un aprendizaje cognitivo situado en el aula - la enseñanza recíproca, el aprendizaje cooperativo, y el modelado participativo - y dar prioridad a la motivación de los alumnos para aprender (como se cita en Echarri, 2009).Todas las anteriores constituyen técnicas para desarrollar un aprendizaje significativo en todas las áreas de la educación, sin embargo, para fines de nuestro argumento también son funcionales para desarrollar aprendizajes significativos dentro del contexto de la Educación Ambiental. Cabe señalar que para Mayer (2004): « [...] la motivación es un aspecto clave en los procesos de enseñanza-aprendizaje» (como se cita en Echarri, 2009), factor que siempre se debe tener en cuenta a la hora de diseñar, proponer y aplicar, con cualquier tipo de estrategia o técnica pedagógica, pero que asimismo influye de forma directa en el manejo, construcción y selección de recursos y material didáctico.

Empero, además de estos elementos, como las estrategias y técnicas, el docente o educador cuenta con una serie de recursos y materiales didácticos que, para ser considerados así, deben tener una intención pedagógica y permitir que los estudiantes interactúen y desarrollen aprendizajes significativos de forma activa y dinámica. Para ilustrar ello, puntualicemos algunas cosas: los materiales didácticos:

[...] logran fortalecer y propiciar esquemas cognitivos más significativos, [...] favorecer el proceso de aprendizaje en los estudiantes, gracias al contacto prácticolúdico con elementos reales que activan el gusto por aprender. [...] son la praxis de 
los conocimientos del maestro, debido que a través de ellos se devela la capacidad del docente para adaptar los contenidos a los procesos y ritmos de los niños; y esto, a su vez, genera en ellos deseos de conocer, preguntarse, explorar e ir más allá de lo que proporciona el maestro (Subrayado ajeno al texto, Manrique y Gallego, 2013, p. 107).

Según Ramírez (Como se cita en Lozano, 2014), los materiales didácticos, también denominados auxiliares didácticos o medios didácticos, pueden ser cualquier tipo de dispositivo diseñado y elaborado con la intención de facilitar un proceso de enseñanza y aprendizaje; es fundamental que estos elementos posibiliten un aprendizaje específico.

Entre sus beneficios, según Rodríguez de Moreno (2004) se pueden destacar: facilitan el logro de los objetivos propuestos, consolidan los conocimientos previos, favorecen la vinculación de conocimientos previamente adquiridos con nuevos conocimientos, estimulan la transferencia de los conocimientos a situaciones diferentes, despiertan el interés y atraen la atención de los estudiantes, proporcionan al estudiante una variedad de experiencias —que facilitan la aplicación de su aprendizaje a situaciones de la vida real—, evitan aquellas actividades y ejercicios que estimulan sólo la retención y la repetición, propician la creatividad (como se cita en Lozano, 2014).

De igual modo: « [...] los materiales abiertos pueden ser de ayuda, [...] trabajar con metodologías activas que contemplen el pensamiento divergente». Echarri, 2009, p. 63), pues permite el manejo o uso de la imaginación o la creatividad, produciendo en el educando resultados novedosos o diversificados, respondiendo así a la heterogeneidad que se encuentra hoy en día en las aulas, por eso se preponderan usar metodologías y recursos de aprendizaje abierto (2009).

Así, se podrá responder adecuadamente a cada necesidad e interés particular del educando, considerando que este aprender de forma diferente, dado que cada niño o persona tiene una forma totalmente diferente de ver el mundo, de comprender, de relacionarse con el entorno y de aprender de él; de esa manera, con una metodología y materiales abiertos se responderá a esa diversidad educativa y formativa, no solamente dentro de la Educación Ambiental sino en toda área de aprendizaje.

No obstante, es adecuado aclarar que:

[...] ningún material, por innovador y completo que sea, es capaz de sustituir a un profesor, al contrario, el maestro siempre será el elemento más significativo en el proceso educativo. El maestro es quien transmite y estructura la información, diseña las situaciones de aprendizaje y diagnostica las habilidades y necesidades de los estudiantes (Lozano, 2014, pp.48-49). 
La pedagogía: el complemento estratégico de la Educación Ambiental | Marcela Andrea Moscoso Casallas | Vilma Aurora Garzón Guerrero |

Finalmente, de acuerdo a Ballester (2002), entre otros recursos tenemos:

[...] recursos delectura — prensa, libros detexto, cuentos, investigación bibliográfica...-; los recursos basados en el trabajo en equipo — debates, dramatizaciones, lluvia de ideas, juegos, juegos de simulación, mesa redonda-; los recursos audiovisuales — DVD, cine, televisión, diapositivas, retroproyector, vídeo, gráficos, mapas conceptuales, esquemas, dibujos, cómics, [...], presentaciones multimedia, internet—; los recursos del medio — trabajo de campo...-. Otros recursos — exposiciones [...] experimentos, construcción de aparatos, crucigramas, museos, huerto escolar, recursos creativos(Como se cita en Echarri, 2009, pp.65-66).

Y, por supuesto, no se puede olvidar el uso de las Tecnologías de la Información y la Comunicación — TIC en Colombia - o Nuevas tecnologías de la información y la Comunicación — NTIC en España—, pues éstas, en palabras de Cabero (2007), dan pie a la tecnología educativa: «su utilización puede implicar la movilización de una diversidad de estrategias y metodologías docentes que favorezcan una enseñanza activa, participativa y constructiva» (como se cita en Echarri, 2009, p. 225). Además, como sostiene Sanmamed (2007), las NTIC o TIC «no sólo son un factor que puede mejorar el proceso educativo, sino que «pueden ayudar a replantear el acto didáctico y a buscar nuevas formas de abordarlo, diseñarlo y desarrollarlo» (como se cita en Echarri, 2009, pp. 218-219). Una forma muy sencilla pero significativa de aplicar tal cosa se da mediante el uso de internet como recurso educativo, por ejemplo, para fomentar la perspectiva holística desde la acción local de la educación o problemas ambientales (Echarri, 2009). Sin embargo, "hacer significativa esta amplitud de información depende del diseño de la actividad instructiva por parte del docente, creando límites o criterios, orientaciones, para la búsqueda de información y para su uso por parte del estudiante» (p. 84).

Se puede finalizar enfatizando en lo siguiente: la Educación Ambiental -EAse puede considerar como el centro estratégico de la conservación, protección y cuidado del medio ambiente, pero para que permita realmente generar un cambio de actitudes y desarrollar valores, acciones, saberes, experiencias, propuestas y reflexiones responsables y amigables con éste, es necesario trabajar desde una pedagogía y didáctica ambiental en las que las estrategias que se apliquen respondan a los requerimientos de la comunidad educativa, pero que, asimismo, tengan en cuenta los componentes interdisciplinares, sociales, contextuales, holísticos, intencionales, transversales, investigativos y teórico-prácticos de la EA. Para ello, el docente cuenta con una serie de herramientas compuesta por estrategias, técnicas y recursos didácticos. Entre algunos de ellos tenemos las TIC, los debates, salidas de campo, investigación, proyectos, presentaciones audiovisuales, uso de material didáctico, entre otros. Cada una de estas estrategias o recursos deben ser abiertas pues, de ese modo, garantizarán una educación y un proceso de enseñanza-aprendizaje inclusivo. Tampoco se puede olvidar la función que tiene el docente dentro de la EA, pues es el encargado de seleccionar, diseñar y aplicar esa intención pedagógica 
en su acto didáctico, en pro de desarrollar aprendizajes significativos, vivenciales, teóricos, prácticos e integrales en el educando; en este caso, la motivación, la creatividad y la participación juegan un papel fundamental como articuladores de saberes, experiencias y aprendizajes ambientales en los que se logre despertar el interés y llamar la atención de los educandos ante el cuidado y la importancia del entorno natural y su relación con el ser humano.

\section{Metodología}

Fue una investigación de carácter cualitativo dado que «desarrolla procesos en términos descriptivos e interpreta acciones, lenguajes, hechos funcionalmente relevantes y los sitúa en una correlación con el más amplio contexto social». (Martínez, 2011, p.11), trabajo que «se orienta a profundizar casos específicos y no a generalizar. Su preocupación no es prioritariamente medir, sino cualificar y describir el fenómeno social a partir de rasgos determinantes» (Lara, 2011). Por consiguiente, fue una investigación de tipo documental o bibliográfica en la que se define «un análisis de la información escrita sobre un determinado tema, con el propósito de establecer relaciones, diferencias, etapas, posturas o estado actual del conocimiento respecto al tema objeto de estudio» (2011).

En ese orden de ideas, se realizó una búsqueda exhaustiva en diferentes bases de datos del Sistema Bibliotecario de la Universidad Minuto de Dios- y material teórico disponible en la web — por ejemplo, de Scielo o Redalyc_-sobre el tema de estudio, a partir del cual se buscó responder la siguiente pregunta de investigación: ¿qué debe tener en cuenta el docente a la hora de aplicar y diseñar estrategias pedagógicas dentro de la Educación Ambiental?

Para responder fue necesario categorizar la información recopilada. Se identificó una categoría principal: Estrategias Pedagógicas en la Educación Ambiental. Esta categoría se subdividió en educación, pedagogía, didáctica, cultura y contexto. Cada una se articuló y se complementó entre sí. La primera subcategoría se centró en la Educación Ambiental como proceso formativo y social. La segunda, se enfatizó en el proceso educativo y sus pautas de aplicación. La tercera, enmarcó todos los componentes de metodología, estrategias, recursos y material didáctico y pedagógico, dentro del contexto ambiental. En cuanto a la cuarta y quinta subcategoría, se trabajaron factores que influían en el proceso formativo de la Educación Ambiental en un ámbito educativo, dinámico y cambiante. Pero, para llevar a cabo dicho procedimiento de categorización, fue necesario, una vez seleccionados los documentos, realizar una lectura detallada de cada uno, para lo cual fue conveniente efectuar con cada uno un Resumen Analítico en Educación-RAE-.

Todo esto se trabajó desde un enfoque hermenéutico, el cual se define, según Martínez (2011), como: «hermenéutica —interpretación- [que] busca descubrir 
los significados de las distintas expresiones humanas, como las palabras, los textos, los gestos, pero conservando su singularidad»(p.12). La parte de interpretación intencional y contextualizada se ejecutó y tiene como finalidad de encontrar pautas teórico-prácticas para la aplicación y selección de estrategias pedagógicas diversas que tiendan o fortalezcan la EA dentro de un contexto educativo formal. Asimismo, la información recopilada se categorizó, analizó e interpretó desde una connotación ambiental, pedagógica y aplicativa.

A su vez, también se cuenta con un enfoque o paradigma sociocrítico, pues se centró la atención en la autorreflexión y construcción de conocimientos a partir de los intereses y las necesidades de la comunidad o grupo social de estudio. Entre las características que se tuvieron en cuenta de este enfoque, de acuerdo a Popkewitz (1988), fueron: "conocer y comprender la realidad como praxis; unir teoría con practica; orientar el conocimiento hacia la emancipación y liberación del ser humano, y proponer la integración de todos los participantes, incluyendo al investigador, en procesos de autorreflexión [...]»(Como se cita en Díaz, et al. 2014, p. 61)

\section{Resultados}

Se identificó que uno de los mayores limitantes de la Educación Ambiental- EA- es la poca claridad y los pocos criterios de selección que existen para elegir o diseñar las estrategias pedagógicas y puedan ser viables, claras y objetivas dentro de un ámbito de educación formal. Igualmente, se observó que es necesario modificar el paradigma educativo, dentro de la aplicación de la EA, por uno que sea de carácter constructivista y significativo para todos los agentes educativos, principalmente para los actores principales dentro del proceso de enseñanza-aprendizaje: docente y discente.

Por último, se concibe a la Educación Ambiental como una estrategia universal, transversal, holística, humana e interdisciplinar, que atiende de forma integral el cuidado y conservación del medio ambiente a partir de la transformación de actitudes, la construcción de saberes, la articulación de experiencias y la planeación de propuestas que fortalezcan la relación hombre-medio. Aquí el docente y los demás actores sociales y educativos son determinantes para la selección, formulación, aplicación, adaptación y contextualización de estrategias pedagógicas que desarrollen aprendizajes significativos y funcionales para el educando.

\section{Conclusiones}

La Educación Ambiental se completa en el momento en que se relaciona con la Pedagogía Ambiental, pues ésta le aporta los componentes estratégicos, teóricos, técnicos, organizativos, didácticos, metodológicos e intencionales de la pedagogía formativa. De esta manera, la EA se convierte en una herramienta y un campo 
interdisciplinar y transversal de formación y generación de cambios en el sujeto, en términos actitudinales, conductuales, cognitivos, comunicativos, sociales y culturales, entorno a su relación, responsabilidad, acciones, conductas y conciencia con el medio ambiente, de tal forma que inicie un proceso de «desaprensión» — de «desaprender»— de aquello que no es amigable ni responsable con el medio ambiente, para lo cual el docente cuenta con un área central de estrategias, métodos, técnicas, materiales y recursos pedagógicos y didácticos denominada didáctica ambiental. Esta didáctica da pautas para la debida aplicación de la EA de modo integral, participativo, significativo, dinámico, vivencial y organizado en un ámbito educativo.

Con la finalidad de seleccionar y aplicar unas estrategias acordes para el uso pedagógico de la EA, es necesario que el docente tenga en cuenta los siguientes aspectos: sus estrategias deben partir del contexto real e inmediato del niño, deben ser contextualizadas y adaptadas, han de estar basadas en un proceso reflexivo, vivencial, lúdico, exploratorio y teórico-práctico; todas la estrategias que seleccione, al igual que sus recursos, evidencian su praxis docente. El uso de estrategias abiertas fomenta una EA inclusiva; las estrategias deben ser generadoras de aprendizajes significativos y motivantes, para desarrollar en el educando el gusto por aprender más allá de lo que el docente le propone en el proceso de enseñanza-aprendizaje.

Conviene subrayar que, entre los mayores cómplices a la hora de promover EA con una intención pedagógica y unas estrategias ambientales diseñadas y definidas, se encuentra la creatividad y la participación por parte del docente y sus estudiantes, puesto que el proceso de aprendizaje se vuelve reciproco, interdependiente, intersubjetivo, critico, reflexivo, significativo e innovador.

Así las cosas, nuestra propuesta final está centrada en la elaboración de una cartilla o catálogo donde se encuentra una serie estrategias pedagógicas, recomendaciones y pautas que debe seguir el docente para desarrollar una Educación Ambiental integral y dinámica en el aula o centro de educación. No obstante, es necesario decir que, ante todo, la regla de oro es siempre partir del contexto real del niño, de sus necesidades e intereses, sólo así se logra construir un vínculo emocional que genere un aprendizaje significativo, funcional y autorreflexivo en el educando. Por este motivo, las estrategias pedagógicas que se encuentra en este recurso o material didáctico constituyen algunos ejemplos a seguir, acompañados de pautas o ideas, para que el docente los adapte y contextualice a sus requerimientos pedagógicos, sociales, culturales y humanos. Es así como se puede ver el uso de las 3R como fundamentación selectiva —reducción-, organizativa y aplicativa —reciclaje y reutilización- dentro de un medio creativo, participativo y pedagógico. De ese modo, este material se convierte en una pauta de selección de estrategias que partan del uso integral de cada recurso o técnicas disponibles ante el proceso de enseñanza-aprendizaje por parte del docente, que permitan desarrollar aprendizajes significativos en el educando. 


\section{Referencias}

Calád, L. (2013). Propuesta de educación para el desarrollo sustentable en el reciclaje y la reutilización de materiales en juegos y juguetes en la educación inicial. Revistas de Estudios y Experiencias en Educación, 12(24), 111-127.

Ceballos, J. (18 de abril de 2013). Reflexiones hacia la pedagogía ambiental como base de la educación. Recuperado de simposioevaluacion.udenar.edu.co:http://simposioevaluacion. udenar.edu.co/wp-content/uploads/2014/10/REFLEXIONES-HACIA-LA-PEDAGOG\%C3\%8DAAMBIENTAL-COMO-BASE-DE-LA-EDUCACI\%C3\%93N.pdf

Díaz, D., Castillo, L., y Díaz, P. (2014). Educación Ambiental y primera infancia: estudio de caso Institución Educativa Normal Superior y Fundación Educadora Carla Cristian del Bajo Cauca [tesis de pregrado]. Bajo Cauca: Universidad de Antioquia.

Echarri, I. (2009). Aprendizaje significativo y Educación Ambiental: aplicaciones didácticas del Museo de Ciencias Naturales de la Universidad de Navarra [tesis doctoral]. Pamplona: Universidad de Navarra.

García, C.,y Galiano, M. (2003). Métodos y estrategias para la Educación Ambiental. Eúphoros, (6), 249-260.

Lara, E. (2011). Fundamentos de investigación. Un enfoque por competencias. México: Alfaomega.

Lozano, J. (2014). El material didáctica de Educación Ambiental y las prácticas al medio ambiente en los centros de educación básica fiscales urbanos del Cantón Tisaleo [tesis de pregrado]. Ambato: Universidad Técnica de Ambato.

Manrique, A., y Gallego, A. (2013). El material didáctico para la construcción de aprendizajes significativos. Revista Colombiana de Ciencias Sociales, 4(1), 101-108.

Martínez, J. (2013). Métodos de investigación cualitativa. Silogismo, 8(1), 1-43.

McMillan, J., y Schumacher, S. (2005). Investigación educativa. Madrid: Pearson Educación.

Rengifo, B., Quitiaquez, L., y Mora, C. (2012). La Educación Ambiental, una estrategia pedagógica que contribuye a la solución de la problemática ambiental en Colombia. Bogotá: XII Coloquio Internacional de Geocrítica.

Rodríguez de Moreno, E. (22 de abril de 2004). ¿Educación ambiental o pedagogía ambiental? Recuperado de pedagogica.edu.co: http://www.pedagogica.edu.co/storage/ps/articulos/ peda07_06arti.pdf

Tovar-Gález, J. (2013). Pedagogía ambiental y didáctica ambiental como fundamentos del currículo para la formación ambiental. Revista Brasileira de Educação, 18(55), 877-1061. 
Recibido: 6 de octubre 2016

Aceptado: 11 de noviembre 2016

Cómo citar: Moscoso, M., y Garzón, V. (2017). La pedagogía:

el complemento estratégico de la Educación Ambiental. Praxis

Pedagógica, 20, 103-122 